\title{
Atezolizumab y bevacizumab en carcinoma hepatocelular irresecable
}

\author{
Rodrigo Arias Z. ${ }^{1}$, Vicente Gándara F. , María Francisca Seydewitz O. ', \\ Francisco Tortorolo C. ${ }^{2}$ y Carlos Benitez G. ${ }^{2}$
}

Atezolizumab plus Bevacizumab in Unresectable Hepatocellular Carcinoma

${ }^{1}$ Escuela de Medicina, Pontificia Universidad Católica de Chile. Santiago, Chile. ${ }^{2}$ Departamento de Gastroenterología Pontificia Universidad Católica de Chile Santiago, Chile.

Recibido 12 de noviembre 2020 Aceptado 20 de noviembre 2020

Correspondencia a:

Dr. Francisco Tortorolo

Departamento Gastroenterología Facultad de Medicina, Pontificia Universidad Católica de Chile.

Santiago, Chile. panchotortorolo@ gmail.com

\section{Pregunta}

En pacientes con carcinoma hepatocelular etapa BCLC C, ¿Es útil el uso de atezolizumab más bevacizumab como primera línea para aumentar la supervivencia global y supervivencia libre de progresión en comparación con el uso de sorafenib?

Para poder responder esta pregunta se revisó el siguiente artículo: Finn RS, Qin S, Ikeda M, et al. Atezolizumab plus Bevacizumab in Unresectable Hepatocellular Carcinoma. N Engl J Med. 2020;382(20):1894-1905'.

\section{Contexto}

El carcinoma hepatocelular $(\mathrm{CHC})$ es el quinto cáncer más común en el mundo, constituyendo el 7\% de todos los cánceres, y la tercera causa de mortalidad relacionada con cáncer según las estimaciones de la Organización Mundial de la Salud². En 2012 se reportaron 782.000 casos a nivel mundial, de los cuales el $83 \%$ fue diagnosticado en regiones de menor desarrollo socioeconómico ${ }^{3}$. La edad de presentación del CHC varía según sexo, zona geográfica y la presencia de factores de riesgo asociados con el desarrollo del cáncer, tales como infecciones por virus hepatitis $\mathrm{B}$ (VHB) y C (VHC), consumo excesivo de alcohol, trastornos metabólicos, fibrosis hepática, entre otras ${ }^{4}$. Además, la incidencia aumenta con la edad, alcanzando el máximo en pacientes en torno a los 70 años ${ }^{5,6}$.

El CHC en etapa temprana es curable mediante la resección, trasplante o ablación ${ }^{7}$. Sin embargo, en la mayoría de los pacientes en que no se realiza tamizaje se presenta en una etapa tardía, en donde la naturaleza agresiva de este cáncer junto con opciones de tratamiento eventualmente limitadas por la presencia de falla hepática o complicaciones de la cirrosis, determinan una baja supervivencia general (17\% a 5 años en Estados Unidos) $)^{8,9}$.

Desde 2008 el tratamiento sistémico de primera línea aprobado para el CHC irresecable es el sorafenib, un inhibidor multiquinasa ${ }^{10-12}$. Esto se debe a que en pacientes con CHC avanzado, se observó una mejoría significativa en la supervivencia global y en el tiempo de progresión radiológica en los pacientes que recibieron sorafenib versus placebo $^{11-13}$.

En relación al perfil de seguridad del sorafenib, estudios han reportado que un $64 \%$ de los pacientes informan un evento adverso (EA) relacionado con el fármaco. La mayoría de los EA relacionados con el fármaco son diarrea, fatiga y reacción cutánea de manos y pies. Además, alrededor de un $9 \%$ de los pacientes experimentan un EA grave relacionado con el medicamento, asimismo, se ha reportado que un $28 \%$ de los pacientes presentan algún EA que resulta en la discontinuación permanente de sorafenib ${ }^{14}$.

Actualmente, existen nuevos fármacos y terapias para el tratamiento del CHC avanzado. Por un lado, los inhibidores de la muerte programada 1 (PD1), que tienen como rol evitar la unión del ligando (PDL1) al receptor PD1 y mostraron un efecto aparentemente benéfico en estudios fase 1 y $2^{15,16}$. Entre otras opciones de tratamiento, se encuentra elanticuerpo monoclonal atezolizumab, que se dirige selectivamente a PDL1 para prevenir la interacción con los receptores PD1 y B71 contrarrestando el efecto de las células reguladoras y favoreciendo la respuesta inmune efectora sobre las células tumorales ${ }^{17}$. Por otro lado, la sobreexpresión del factor de crecimiento endotelial vascular (VEGF), se ha relacionado con el desarrollo y la progresión de $\mathrm{CHC}^{18}$. Las terapias anti-VEGF mediadas por bevacizumab inhiben la angiogénesis y crecimiento tumoral ${ }^{19}$, ya que reduce la actividad inmunoreguladora dentro del tumor y de su microambiente $^{20}$. Además, bevacizumab podría eventualmente potenciar el efecto de atezolizumab, revirtiendo el efecto supresor mediado por VEGF y potenciando la migración celular y actividad de los linfocitos $\mathrm{T}^{21}$.

Como se mencionó anteriormente, hasta ahora el tratamiento estándar en fases avanzadas se limita al 
uso de sorafenib, el cual prolonga la supervivencia global (SG) aunque se asocia a efectos adversos frecuentes ${ }^{11-14}$. Por otro lado, el uso de atezolizumab y bevacizumab para el tratamiento de CHC etapa BCLC C, podría ser una opción terapéutica apropiada para mejorar la supervivencia general comparado con sorafenib.

A continuación, se analiza un estudio que tiene como objetivo evaluar la SG y la supervivencia libre de progresión (SLP), en pacientes con CHC irresecable, comparando el uso de terapia con atezolizumab más bevacizumab respecto al estándar de tratamiento actual con sorafenib.

\section{Métodos}

\section{Características generales}

Participantes. Se enrolaron 501 participantes, de 18 años o más, con diagnóstico de $\mathrm{CHC}$ no resecable, con al menos una lesión medible según Response Evaluation Criteria in Solid Tumors (RECIST 1.1), no candidato a terapia curativa o bien que haya sido tratado de forma regional con progresión posterior. Fueron criterios de inclusión: a) no haber recibido terapia sistémica previa para $\mathrm{CHC}$; b) estado funcional de 0 o 1 en la escala del Eastern Cooperative Oncology Group (ECOG); c) ser clasificados como Child-Pugh A; d) ausencia de infección por virus de inmunodeficiencia humana; e) presentar una carga viral $<500 \mathrm{U} / \mathrm{mL}$ en casos de infección por VHB y haber recibido tratamiento antiviral por lo menos 14 días previos a la inclusión; f) resolución de los efectos tóxicos agudos de terapias previas, con la excepción de alopecia; g) tanto mujeres como hombres debían estar de acuerdo con permanecer abstinente o usar métodos anticonceptivos con una tasa de fracaso de $<1 \%$ anual durante el estudio y temporalidades establecidas posterior a la última dosis de cada medicamento según la duración de su potencial teratogénico; h) debían presentar función orgánica y hematológica adecuadas, esta últimas definidas como: transaminasas y fosfatasas alcalinas $<5$ veces el valor normal, bilirrubina total $<3$, albúmina $\geq 2,8$, índice internacional randomizado (INR) $<2$, clearance creatinina $\geq 50 \mathrm{~mL} / \mathrm{min}$ o creatinina plasmática $\leq 1,5$ veces el valor normal, proteinuria $<1$ g en 24 horas, sin neutropenia o linfopenia, plaquetas $>75.000 \mathrm{U} /$ mcL y $\mathrm{Hb}>9 \mathrm{~g} / \mathrm{dL}$. Por otro lado, se excluyeron del estudio a pacientes que presentaran: a) enfermedades autoinmunes (con la excepción de hipotiroidismo, diabetes mellitus tipo 1 y cutáneas leves sin compromiso sistémico, todas en tratamiento y controladas); b) coagulopatías o tratamiento con anticoagulantes y/o antiplaquetarios (excepto ácido acetil salicílico); c) várices esofágicas o gástricas de alto riesgo no tratadas o incompletamente tratadas, diagnosticadas por endoscopía digestiva alta (EDA) durante los 6 meses previos a la inclusión; d) coinfección de VHB $\mathrm{y}$ virus hepatitis $\mathrm{C}$ (VHC); e) ascitis moderada o severa; f) encefalopatía hepática; g) uso de inmunosupresores, inmunoterapia, inmunoestimulantes o radioterapia reciente previa; h) $\mathrm{CHC}$ fibrolamelar, sarcomatoide, o coexistencia de colangiocarcinoma; i) angina inestable, arritmia inestable o enfermedad cardiovascular significativa (como insuficiencia cardíaca clase II o más en la escala de la New York Heart Association, infarto agudo al miocardio o accidente cerebrovascular) presente durante los 3 meses previos al inicio del estudio; j) hipertensión arterial mal controlada, historia de crisis hipertensivas o encefalopatía hipertensiva; entre otros.

Intervención. Los participantes fueron aleatoriamente asignados a dos grupos, en una relación de 2:1, y estratificado según: a) región geográfica (Asia excluyendo a Japón versus el resto del mundo); b) presencia vs. ausencia de invasión macrovascular o compromiso extrahepático; c) nivel de alfafetoproteína basal $(<400$ versus $\geq 400 \mathrm{ng} / \mathrm{mL})$; d) estado funcional según la escala del ECOG (0 versus 1). El primer grupo fue compuesto por 336 participantes que recibieron $1200 \mathrm{mg}$ de atezolizumab más 15 mg por kilogramo de peso corporal de bevacizumab intravenoso, cada 3 semanas. El segundo grupo consistió en 165 participantes que recibieron $400 \mathrm{mg}$ de sorafenib vía oral dos veces al día. Se determinó que se detendría la administración del tratamiento con la presentación de efectos adversos inaceptables o una vez identificada, por parte de los investigadores, una pérdida en el beneficio clínico. Los participantes y tratantes no fueron ciegos a la intervención.

\section{Objetivos}

Objetivos primarios. $\mathrm{SG}$, definida como el tiempo desde aleatorización hasta la muerte por cualquier causa; y SLP, definida como el tiempo desde aleatorización hasta progresión de enfermedad o muerte por cualquier causa, el que ocurra primero. Definen progresión de enfermedad utilizando RECIST $1.1 \mathrm{y}$ mRECIST, sin embargo la categorización final utilizada en los resultados principales es según RECIST 1.1.

Objetivos secundarios. Se definieron los siguientes desenlaces secundarios: a) respuesta objetiva (RO), definida como el porcentaje de pacientes con una respuesta completa o parcial confirmada; b) duración de respuesta, definida como el tiempo desde la primera respuesta completa o parcial documentada hasta la progresión de enfermedad o muerte; c) tiempo al deterioro de calidad de vida, funcionamiento físico 
o actividades cotidianas, definiendo deterioro como una disminución de 10 o más puntos en los cuestionarios de calidad de vida en cáncer del European Organization for Research and Treatment of Cancer (EORTC QLQ-C30), mantenida en dos evaluaciones consecutivas o seguida por muerte por cualquier causa durante las 3 semanas siguientes; y d) seguridad y perfil de efectos adversos, incluyendo tipo, frecuencia y severidad de estos según los National Cancer Institute (NCI) Common Terminology Criteria for Adverse Events (version 4.0).

\section{Evaluación de validez interna}

Diseño. Estudio clínico fase III, aleatorizado, abierto para los participantes e investigadores, multicéntrico, incluyendo 111 centros en los siguientes 17 países: Alemania, Australia, Canadá, China, España, Estados Unidos, Francia, Hong Kong, Italia, Japón, Polonia, Reino Unido, República Checa, República de Corea, Rusia, Singapur y Taiwán.

Aleatorización. Esta fue realizada mediante la asignación por sistema de voz interactivo o sistema de respuesta basado en la web, en una relación 2:1 (atezolizumab-bevacizumab y sorafenib, respectivamente), con un método de bloques permutado y estratificación de las variables descritas anteriormente.

Grupos similares en variables conocidas. La distribución de los pacientes entre ambos grupos de tratamiento fue equilibrada en relación a edad, sexo, región geográfica, estado funcional, clasificación Child-Pugh, niveles de alfafetoproteína (AFP) basal, invasión macrovascular (IMV), compromiso extrahepático $(\mathrm{CEH})$, presencia de várices, causa de $\mathrm{CHC}$, y terapia local previa para $\mathrm{CHC}$. Además, la aleatorización se realizó de forma estratificada por las variables especificadas anteriormente en el apartado Intervención.

Ciego. Ensayo abierto, tanto los participantes como los investigadores no fueron ciegos a la intervención. La entidad independiente que realizó el análisis imagenológico durante el seguimiento fue ciega.

Seguimiento. La evaluación de la progresión de enfermedad y respuesta a tratamiento se realizó mediante tomografías computadas o resonancias magnéticas basales, cada 6 semanas hasta la semana 54 de tratamiento, y luego cada 9 semanas hasta el término de la intervención. El análisis imagenológico fue realizado de manera ciega por una entidad independiente. Para la SLP se consideró RECIST 1.1, en cambio en el análisis de la RO y duración de respuesta se utilizó RECIST 1.1 y mRECIST. Los desenlaces secundarios reportados por los participantes fueron evaluados usando los cuestionarios EORTC QLQC30, logrando un cumplimiento del $93 \%$ a la semana 51 de tratamiento, y $80 \%$ al término de la intervención. La seguridad de las intervenciones fue evaluada mediante la medición regular de signos vitales y exámenes de laboratorio. Por último, la incidencia, tipo y severidad de los efectos adversos presentados, fueron evaluadas de manera estandarizada aplicando los NCI Common Terminology Criteria for Adverse Events (versión 4.0). En cuanto a la duración del seguimiento, ésta tuvo una mediana de 8,6 meses $(8,9$ y 8,1 meses en los grupos atezolizumab-bevacizumab y sorafenib, respectivamente).

Escenario. Participantes provenientes 111 centros, de 17 países en Asia, Europa, y América del Norte (EE.UU. y Canadá).

Tipo de análisis de resultados. El efecto de las intervenciones sobre SG y SLP fue evaluado comparando ambos grupos de tratamiento mediante el Test Log-Rank, y a lo largo del seguimiento por medio de la estimación de hazard ratios (HR) utilizando modelos de regresión de Cox. Se aplicaron curvas de Kaplan-Meier a SG, SLP, duración de respuesta y tiempo al deterioro de variables reportadas por participantes. La variable secundaria RO fue comparada entre grupos utilizando el Test de CochranMantel-Haenszel. Todos los análisis de eficacia, con la excepción de los análisis por subgrupos, fueron estratificados por las mismas variables usadas en la aleatorización (excluyendo estado funcional). Fue por intención de tratar, excepto en el análisis de seguridad donde se incluyeron únicamente a los pacientes que recibieron al menos una dosis de tratamiento (329 y 156 participantes en los grupos atezolizumab más bevacizumab y sorafenib, respectivamente).

Interrupción precoz por beneficio. Se calculó un tamaño muestral de 480 participantes, entre ellos 312 muertes para proveer $80 \%$ de potencia para HR de 0,71 en SG a favor de atezolizumab-bevacizumab. Los niveles de significancia bidireccionales fueron 0,048 para SG y 0,002 para SLP, controlado con procedimiento secuencial de Holm. Se planificó realizar un análisis primario de SLP, dos análisis interinos y un análisis final de SG. La realización del análisis primario y el primer análisis interino se debía llevar a cabo al llegar a 308 eventos de progresión de enfermedad o muerte. El 29 de agosto de 2019, se llegó a un total de 306 eventos, incluyendo 161 muertes y se realizó el primer análisis interino, determinando un nivel de significancia bidireccional para SG de 0,0033. Luego de esto, el estudio se interrumpió precozmente por beneficio. No se esclarecen los criterios utilizados para tomar esta decisión. 
Resultados. Las tasas de SG y SLP fueron significativamente mayores en el grupo atezolizumabbevacizumab en comparación con el grupo sorafenib. Las tasas de SG a 6 y 12 meses fueron de 84,8\% (IC $95 \% 80,9-88,7$ ) y $67,2 \%$ (IC 95\% 61,3-73,1), respectivamente en el grupo atezolizumab-bevacizumab, y $72.2 \%$ (IC 95\% 65,1-79,4) y 54,6\% (IC 95\% 45,264), respectivamente en el grupo sorafenib. No se alcanzó la mediana de supervivencia global en el grupo atezolizumab-bevacizumab durante el tiempo de observación. La mediana de SLP en el grupo atezolizumab-bevacizumab y sorafenib, fue de 6,8 (IC 95\% 5,7-8,3) y 4,3 (IC 95\% 4,0-5,6) meses, respectivamente.

En el análisis por subgrupos de SG, destaca un hazard ratio (HR) mayor a 1 en los participantes con $\mathrm{CHC}$ etapa $\mathrm{B}$, pero con un bajo número de participantes y de eventos reportados para esta etapa (9 para el grupo atezolizumab-bevacizumab y 4 para el grupo sorafenib, de un total de 78 participantes). Asimismo, en los subgrupos estratificados por grado de progresión de la enfermedad (como invasión macrovascular o compromiso extrahepático), se observó un beneficio más evidente en aquellos participantes con $\mathrm{CHC}$ de etapas más avanzadas en comparación con los participantes con $\mathrm{CHC}$ de etapas iniciales, con resultados al límite de la significancia estadística. No obstante, el número de participantes con $\mathrm{CHC}$ en etapas más avanzadas fue mayor y en el tiempo de seguimiento se alcanzaron más eventos. En cuanto a etiología de CHC, el subgrupo de etiología no viral no demostró diferencias estadísticamente significativas de SG entre ambas ramas con un HR de 0,91 (IC 95\%, 0,52-1,60). Por el contrario, en el subgrupo de $\mathrm{CHC}$ secundario a VHB y VHC se observó una clara significancia estadística a favor del grupo atezolizumab-bevacizumab.

En relación a la SLP, se evidenciaron diferencias en los subgrupos con $\mathrm{CHC}$ de etapas menos avanzadas en cuanto IMV y CEH (cada uno por separado), pero sin pesquisar diferencias estadísticamente significativas en los subgrupos con AFP $>400$ y BCLC B, pese a estas ambos subrepresentados en comparación a sus respectivas contrapartes.

En relación a los desenlaces secundarios, el tiempo a deterioro ( $\mathrm{TaD}$ ) de calidad de vida, funcionamiento físico y actividades cotidianas, fue de 11,2 (IC 95\% 6,0-NE), 13,1 (IC 95\% 9,7-NE) y 9,1 meses (IC 95\% 6,5-NE), respectivamente para el grupo atezolizumab-bevacizumab, y 3,6 (IC 95\% 3,0-7,0), 4,9 (IC 95\% 3,5-6,2) y 3,6 (IC 95\% 2,2-6,0) meses, respectivamente para el grupo sorafenib. El HR para $\mathrm{TaD}$ de calidad de vida, funcionamiento físico y actividades cotidianas fue de 0,63 (IC 95\% 0,46-0,85), 0,53 (IC 95\% 0,39-0,73) y 0,62 (IC 95\% 0,46-0,84), respectivamente a favor del grupo atezolizumabbevacizumab.
Se reportaron eventos adversos (EA) de cualquier grado en el $98,2 \%$ de los participantes del grupo atezolizumab-bevacizumab y en el $98,7 \%$ de los participantes del grupo sorafenib. La tasa de EA serios en el grupo atezolizumab-bevacizumab y en el grupo sorafenib fue de $38 \%$ y $30,8 \%$, respectivamente, sin establecer el grado de significancia estadística. Los EA más frecuentes en el grupo atezolizumab-bevacizumab fueron hipertensión arterial, fatiga, proteinuria, elevación de SGOT, prurito y diarrea, en orden decreciente, los que en su mayoría fueron leves. En cambio, en el grupo sorafenib los EA más frecuentes fueron diarrea, síndrome de eritrodisestesia palmoplantar, hipertensión arterial, disminución del apetito y fatiga, en orden decreciente. En ambos grupos el evento adverso grado 3-4 más frecuentemente reportado fue hipertensión arterial, con tasas de $10,3 \%$ en grupo atezolizumab-bevacizumab y $9 \%$ en el grupo sorafenib). El 15\% de los participantes del grupo atezolizumab-bevacizumab suspendió la terapia por un evento adverso, en comparación con un $10 \%$ en el grupo sorafenib. La incidencia de hemorragia digestiva alta (HDA) en el grupo atezolizumab-bevacizumab y en el grupo sorafenib fue de $7 \%$ y $4,5 \%$, respectivamente, sin establecer el grado de significancia estadística, lo que es consistente con la incidencia de HDA reportada en otros estudios en relación al uso de bevacizumab para CHC.

Los principales resultados se resumen en la Tabla 1, 2, 3 y 4. El análisis de seguridad y efectos adversos se detalla en la Tabla 5 y 6.

\section{Comentarios}

\section{Generales}

El presente estudio busca establecer la efectividad de la terapia con atezolizumab-bevacizumab en comparación con la terapia de primera línea basada en sorafenib, en pacientes con $\mathrm{CHC}$ no resecable sin terapia sistémica previa. Este estudio es relevante, porque la terapia con sorafenib, pese a ser el gold standard, tiene un efecto relativamente modesto en SG y se ha asociado a una tasa considerable de EA. Por lo tanto, ha surgido la necesidad de explorar opciones terapéuticas que permitan prolongar la SG y SLP, idealmente con menores tasas de efectos adversos asociadas.

Este estudio demuestra que la terapia con atezolizumab-bavacizumab se asoció a SG y SLP significativamente mayores en comparación a la terapia con sorafenib, en un grupo seleccionado de pacientes con $\mathrm{CHC}$ no resecable y función hepática conservada. Además, la terapia combinada prolongó el tiempo al deterioro de la calidad de vida y el funcionamiento, en comparación con los reportes de los pacientes que utilizaron sorafenib. 


\section{Medicina Basada en la evidencia en Gastroenterología}

Tabla 1. Resultados de los desenlaces supervivencia global y supervivencia libre de progresión

\begin{tabular}{|c|c|c|c|c|c|c|c|c|c|}
\hline $\begin{array}{l}\text { Desenlaces } \\
\text { primarios }\end{array}$ & $\begin{array}{c}\mathrm{N}^{0} \text { eventos/ } \\
\mathrm{N}^{\mathbf{0}} \\
\text { participantes } \\
(\%)\end{array}$ & $\begin{array}{c}\text { Super- } \\
\text { vivencia } \\
\text { mediana, } \\
\text { meses } \\
\text { (IC 95\%) }\end{array}$ & $\begin{array}{c}\text { Super- } \\
\text { viviencia } \\
\text { a } 6 \text { meses, } \\
\% \\
\text { (IC } 95 \% \text { ) }\end{array}$ & $\begin{array}{c}\text { Super- } \\
\text { viviencia } \\
\text { a } 12 \text { meses, } \\
\% \\
\text { (IC } 95 \%)\end{array}$ & $\begin{array}{c}\text { HR } \\
\text { (IC 95\%) }\end{array}$ & $\begin{array}{c}\text { RR } \\
(\text { IC 95\%) }\end{array}$ & $\begin{array}{c}\text { RRR } \\
(\text { IC 95\%) }\end{array}$ & $\begin{array}{c}\text { RRA } \\
(\text { IC 95\%) }\end{array}$ & $\begin{array}{c}\text { NNT } \\
\text { (IC 95\%) }\end{array}$ \\
\hline \multicolumn{10}{|l|}{$\begin{array}{l}\text { Supervivencia } \\
\text { global (SG) }\end{array}$} \\
\hline $\begin{array}{l}\text { Atezolizumab- } \\
\text { bevacizumab }\end{array}$ & $\begin{array}{c}96 / 336 \\
(28,6)\end{array}$ & $\mathrm{NE}$ & $\begin{array}{c}84,8 \\
(80,9-88,7)\end{array}$ & $\begin{array}{c}67,2 \\
(61,3-73,1)\end{array}$ & $\begin{array}{c}0,58 \\
(0,42-0,79) \\
\mathrm{P}<0,001\end{array}$ & $\begin{array}{c}0,73 \\
(0,56-0,93)\end{array}$ & $\begin{array}{c}0,27 \\
(0,07-0,44)\end{array}$ & $\begin{array}{c}10,82 \% \\
(1,94-19,56)\end{array}$ & $\begin{array}{c}9 \\
(5-51)\end{array}$ \\
\hline Sorafenib & $\begin{array}{l}65 / 165 \\
(39,4)\end{array}$ & $\begin{array}{c}13,2 \\
(10,4-\mathrm{NE})\end{array}$ & $\begin{array}{c}72,2 \\
(65,1-79,4)\end{array}$ & $\begin{array}{c}54,6 \\
(45,2-64,0)\end{array}$ & & & & & \\
\hline \multicolumn{10}{|c|}{$\begin{array}{l}\text { Supervivencia libre } \\
\text { de progresión (SLP) }\end{array}$} \\
\hline $\begin{array}{l}\text { Atezolizumab- } \\
\text { bevacizumab }\end{array}$ & $\begin{array}{c}197 / 336 \\
(58,6)\end{array}$ & $6,8(5,7-8,3)$ & 54,5 & & $\begin{array}{c}0,59 \\
(0,47-0,76) \\
\mathrm{P}<0,001\end{array}$ & $\begin{array}{c}0,89 \\
(0,77-1,02)\end{array}$ & $\begin{array}{c}0,11 \\
(-0,02- \\
0,23)\end{array}$ & $\begin{array}{c}7,43 \% \\
(-1,2-16,54)\end{array}$ & $\begin{array}{c}13 \\
(6 a-83)\end{array}$ \\
\hline Sorafenib & $\begin{array}{c}109 / 165 \\
(66,1)\end{array}$ & $\begin{array}{c}4,3 \\
(4,0-5,6)\end{array}$ & 37,2 & & & & & & \\
\hline
\end{tabular}

HR: hazard ratio; RR: riesgo relativo; RRR: reducción del riesgo relativo; RRA: reducción del riesgo absoluto; NNT: número necesario a tratar.

Tabla 2. Resultados de los desenlaces respuesta objetiva y duración de respuesta

\begin{tabular}{|c|c|c|c|c|}
\hline Desenlaces secundarios objetivos & $\begin{array}{c}\mathrm{N}^{0} \text { eventos/ } \\
\mathrm{N}^{0} \text { participantes }(\%)\end{array}$ & Delta \% (P value) & RR (IC 95\%) & NNT (IC 95\%) \\
\hline \multicolumn{5}{|l|}{ Respuesta objetiva (RO) RECIST 1.1} \\
\hline Atezolizumab - bevacizumab & $89 / 326(27,3)$ & $15,4(<0,001)$ & $2,28(1,45-3,61)$ & $7(4-11)$ \\
\hline Sorafenib & $19 / 159(11,9)$ & & & \\
\hline \multicolumn{5}{|l|}{ Respuesta objetiva (RO) mRECIST } \\
\hline Atezolizumab - bevacizumab & $108 / 325(33,2)$ & $19,9(<0,001)$ & $2,5(1,63-3,83)$ & $5(4-8)$ \\
\hline Sorafenib & $21 / 158(13,3)$ & & & \\
\hline \multicolumn{5}{|l|}{ Duración de respuesta ( $>6$ meses) } \\
\hline Atezolizumab - bevacizumab & $(87,6)$ & 28,5 & $1,48(1,4-1,57)$ & $4(3-4)$ \\
\hline Sorafenib & $(59,1)$ & & & \\
\hline
\end{tabular}

RR: riesgo relativo; NNT: número necesario a tratar.

Tabla 3. Resultados de desenlaces secundarios reportados por los pacientes

\begin{tabular}{|lcc|}
\hline Desenlaces secundarios subjetivos & TaD mediana, meses (IC 95\%) & HR (IC 95\%) \\
Tiempo a deterioro (TaD) de calidad de vida & $11,2(6,0-\mathrm{NE})$ & $0,63(0,46-0,85)$ \\
Atezolizumab - bevacizumab & $3,6(3,07,0)$ & \\
Sorafenib & & \\
TaD de funcionamiento físico & $13,1(9,7-\mathrm{NE})$ & $0,53(0,39-0,73)$ \\
Atezolizumab - bevacizumab & $4,9(3,5-6,2)$ & \\
Sorafenib & & $0,62(0,46-0,84)$ \\
TaD de actividades cotidianas & $9,1(6,5-\mathrm{NE})$ & \\
Atezolizumab - bevacizumab & $3,6(2,2-6,0)$ & \\
Sorafenib & & \\
\hline
\end{tabular}

HR: hazard ratio. 


\section{Medicina Basada en la evidencia en Gastroenterología}

Tabla 4. Comparación entre análisis realizado por comité independiente vs. investigadores

\begin{tabular}{|c|c|c|}
\hline Intervención & SLP mediana, meses (IC 95\%) & RO, $n^{0}$ eventos $/ n^{\circ}$ total $(\%)$ \\
\hline \multicolumn{3}{|l|}{ Análisis por comité independiente } \\
\hline Atezolizumab - bevacizumab & $6,8(5,7-8,3)$ & $89 / 326(27,3)$ \\
\hline Sorafenib & $4,3(4,0-5,6)$ & $19 / 159(11,9)$ \\
\hline \multicolumn{3}{|l|}{ Análisis por investigadores } \\
\hline Atezolizumab - bevacizumab & $7,1(5,7-8,4)$ & $86 / 336(25,6)$ \\
\hline Sorafenib & $2,9(2,8-4,2)$ & $9 / 165(5,5)$ \\
\hline
\end{tabular}

SLP: supervivencia libre de progresión; RO: respuesta objetiva.

Tabla 5. Efectos adversos por cualquier causa

\begin{tabular}{|c|c|c|}
\hline \multirow[t]{2}{*}{ Variable } & $\begin{array}{l}\text { Atezolizumab-bevacizumab } \\
\qquad(\mathbf{N}=329)\end{array}$ & $\begin{array}{l}\text { Sorafenib } \\
(\mathbf{N}=156)\end{array}$ \\
\hline & \multicolumn{2}{|c|}{ número (\%) } \\
\hline Pacientes con un evento adverso de cualquier causa & $323(98,2)$ & $154(98,7)$ \\
\hline Evento grado 3 o 4 & $186(56,5)$ & $86(55,1)$ \\
\hline Evento grado 5 & $15(4,6)$ & $9(5,8)$ \\
\hline Evento adverso serio & $125(38,0)$ & $48(30,8)$ \\
\hline Evento adverso que llevó a suspensión de cualquier droga del estudio & $51(15,5)$ & $16(10,3)$ \\
\hline Suspensión de atezolizumab-bevacizumab & $23(7,0)$ & - \\
\hline $\begin{array}{l}\text { Evento adverso que llevó a la modificación de la dosis o interrupción de cualquier } \\
\text { droga del estudio }\end{array}$ & $163(49,5)$ & $95(60,9)$ \\
\hline Interrupción de cualquier tratamiento del estudio & $163(49,5)$ & $64(41,0)$ \\
\hline Modificación de la dosis de sorafenib & - & $58(37,2)$ \\
\hline
\end{tabular}

Los números representan el grado de severidad de los EA, siendo más severos a mayor grado. Los eventos grado 5 en el grupo atezolizumabbevacizumab incluyeron: hemorragia gastrointestinal (en 3 pacientes), neumonía (en dos pacientes), empiema, perforación de úlcera gástrica, función hepática anormal, injuria hepática, síndrome de falla orgánica múltiple, hemorragia esofágica variceal, hemorragia subaracnoidea, distrés respiratorio, sepsis, paro cardiorrespiratorio (en un paciente). Los eventos grado 5 en el grupo sorafenib incluyeron muerte (en dos pacientes), cirrosis hepática (en dos pacientes), paro cardiorrespiratorio, falla cardiaca, deterioro de salud física en general, hepatitis E (en un paciente) y hemorragia peritoneal (en un paciente).

Se evidenció una mayor tasa de EA serios y de EA que llevaron a la suspensión del protocolo de tratamiento en el grupo atezolizumab-bevacizumab en comparación al grupo sorafenib, pese a que no se realizó un análisis estadístico al respecto. Cabe mencionar, que si bien se registraron EA graves con el uso de atezolizumab-bavacizumab no se observaron EA nuevos o inesperados.

\section{Riesgo de sesgo}

El estudio se realizó siguiendo un diseño metodológico aleatorizado, con distribución equilibrada y estratificada de los participantes a ambos grupos de tratamiento según variables definidas. Pese a que el estudio fue abierto para investigadores y participantes, el desenlace SG es una variable objetiva que no se vería influenciada por falta de ciego. Por otro lado, si bien la SLP pudiese haberse visto afectada, su evaluación por criterios definidos según RECIST 1.1 y por un comité independiente ciego, permite disminuir el riesgo de sesgo en su adjudicación.

Sin embargo, es relevante considerar que existieron diferencias entre los investigadores y el comité independiente respecto a la estimación de SLP utilizando los mismos criterios RECIST 1.1. Los investigadores estimaron que el grupo sorafenib progresaba antes que lo considerado por el comité independiente (poner esas diferencias en números). Esto, pudiese haber influido en forma decisiva en que el tiempo de uso efectivo de terapia con sorafenib haya sido considerablemente menor que la terapia con atezolizumabbevacizumab (mediana de 2.8 versus 7,4 meses, 


\section{Medicina Basada en la evidencia en Gastroenterología}

Tabla 6. Efectos adversos con una incidencia de más de $10 \%$ en cualquier grupo

\begin{tabular}{|c|c|c|c|c|}
\hline Evento & $\begin{array}{c}\text { Atezolizumab- } \\
\text { bevacizumab } \\
(\mathrm{N}=329) \\
\text { Cualquier grado } \\
\text { n }(\%)\end{array}$ & $\begin{array}{c}\text { Grado } 3 \text { o } 4 \\
\text { n }(\%)\end{array}$ & $\begin{array}{c}\text { Sorafenib } \\
(\mathrm{N}=156) \\
\text { Cualquier grado } \\
\text { n (\%) }\end{array}$ & $\begin{array}{c}\text { Grado } 3 \text { o } 4 \\
\text { n (\%) }\end{array}$ \\
\hline Hipertensión & $98(29,8)$ & $50(15,2)$ & $38(24,4)$ & $19(12,2)$ \\
\hline Fatiga & $67(20,4)$ & $8(2,4)$ & $29(18,6)$ & $5(3,2)$ \\
\hline Proteinuria & $66(20,1)$ & $10 \quad(3,0)$ & $11(7,1)$ & $1(0,6)$ \\
\hline Elevación de SGOT & $64(19,5)$ & $23(7,0)$ & $26(16,7)$ & $8(5,1)$ \\
\hline Prurito & $64(19,5)$ & 0 & $15(9,6)$ & 0 \\
\hline Diarrea & $62(18,8)$ & $6(1,8)$ & $77(49,4)$ & $8(5,1)$ \\
\hline Disminución del apetito & $58(17,9)$ & $4 \quad(1,2)$ & $38(24,4)$ & $6 \quad(3,8)$ \\
\hline Pirexia & $59(17,9)$ & $4 \quad(1,2)$ & $15(9,6)$ & $2(1,3)$ \\
\hline Elevación de SGPT & $46(14,0)$ & $12(3,6)$ & $14(9,0)$ & $2(1,3)$ \\
\hline Constipación & $44(13,4)$ & 0 & $22(14,1)$ & 0 \\
\hline Incremento de bilirrubina sérica & $43(13,1)$ & $8 \quad(2,4)$ & $22(14,1)$ & $10(6,4)$ \\
\hline Rash & $41(12,5)$ & 0 & $27(17,3)$ & $4 \quad(2,6)$ \\
\hline Dolor abdominal & $40(12,2)$ & $4(1,2)$ & $27(17,3)$ & $4 \quad(2,6)$ \\
\hline Náuseas & $40(12,2)$ & $1 \quad(0,3)$ & $25(16,0)$ & $1(0,6)$ \\
\hline Tos & $39(11,9)$ & 0 & $15(9,6)$ & $1(0,6)$ \\
\hline Reacción relacionada a la infusión & $37(11,2)$ & $8(2,4)$ & 0 & 0 \\
\hline Baja de peso & $37(11,2)$ & 0 & $15(9,6)$ & $1(0,6)$ \\
\hline Disminución de plaquetas & $35(10,6)$ & $11(3,3)$ & $18(11,5)$ & $2(1,3)$ \\
\hline Epistaxis & $34(10,3)$ & 0 & $7(4,5)$ & $1(0,6)$ \\
\hline Astenia & $22(6,7)$ & $1(0,3)$ & $21(13,5)$ & $4(2,6)$ \\
\hline Alopecia & $4(1,2)$ & 0 & $22(14,1)$ & 0 \\
\hline Síndrome de eritrodisestesia palmo-plantar & $3(0,9)$ & 0 & $75(48,1)$ & $13(8,3)$ \\
\hline
\end{tabular}

SGOT: transaminasa glutámico oxalacética; SGPT: transaminasa glutámico pirúvica.

respectivamente), concordante con la SLP estimada por los investigadores en comparación con el comité independiente (mediana de 2,9 y 7,1 meses, respectivamente), como se representa en la Tabla 4. Esto podría haber impactado en la efectividad reportada de la terapia con sorafenib de haber sido implementada por periodos equivalentes a la terapia con atezolizumabbevacizumab. No obstante lo anterior, el beneficio global del tratamiento con atezolizumab-bevacizumab es muy significativo en comparación a sorafenib y el estudio presenta otras fortalezas considerables, por lo que los elementos cuestionables descritos no invalidan los resultados observados.

Es relevante considerar que en el grupo sorafenib $11,5 \%$ de los participantes abandonaron el estudio, seis de ellos no recibieron el tratamiento y los trece restantes abandonaron una vez iniciado el protocolo. En cambio, el grupo atezolizumab-bevacizumab perdió el 3,5\% de sus participantes, cinco de ellos no recibieron el tratamiento y los siete restantes lo abandonaron una vez iniciado el protocolo. Dado que el análisis de los resultados fue por intención de tratar, el grupo sorafenib fue por consiguiente el más perjudicado al evaluar la efectividad de la terapia.

En el análisis de SG, destaca que los participantes con CHC estadío B de BCLC, sin IMV, sin CEH y AFP mayor a 400, no presentaron diferencias estadísticamente significativas, con intervalos de confianza muy amplios que sobrepasan el 1. Esto podría ser explicado por el menor número de participantes en dichos subgrupos y de eventos observados, resultando en una menor potencia. A modo de ejemplo, 
el subgrupo con CHC estadío B de BCLC estuvo compuesto por 78 participantes versus 409 con $\mathrm{CHC}$ estadío C de BCLC. Estos subgrupos podrían beneficiarse de un periodo de seguimiento más prolongado para lograr una potencia adecuada, en consideración de la menor gravedad y la mayor sobrevida asociadas a estas etapas de la enfermedad.

El mismo fenómeno se observa en relación al subgrupo de etiología no viral, en comparación con el subgrupo de etiologías VHB y VHC en conjunto, abriendo espacio para nuevos estudios sobre este subgrupo en específico.

\section{Relevancia de los datos}

Los datos son relevantes, ya que entregan una nueva opción terapéutica basada en atezolizumab y bevacizumab que reporta mejores resultados en SG y SLP en pacientes con CHC irresecable, en comparación al actual estándar de terapia. En base a ello, es posible que la combinación atezolizumab y bevacizumab se transforme en el nuevo estándar de tratamiento para esta patología en hepatopatía de origen no autoinmune. No obstante, como ya se señaló previamente, es pertinente realizar nuevos estudios con los subgrupos menos representados en el ensayo clínico analizado, particularmente en aquellos de etiología no viral.

\section{Aplicabilidad externa}

El estudio se realizó en una población con función hepática preservada (Child-Pugh A), con un riesgo relativamente bajo de HDA variceal, sin encefalopatía hepática ni ascitis importantes, y sin comorbilidades relevantes, por lo que los resultados observados serían aplicables en una población seleccionada. Es por esto, que no se pueden extrapolar los resultados observados a grupos que no cumplan con estas características. Además, el 70\% de los $\mathrm{CHC}$ fueron de etiología viral, lo que resta certeza sobre un claro beneficio sobre el estándar con la terapia con atezolizumab-bevacizumab, en poblaciones con cirrosis secundaria a otras etiologías, que serían las más frecuentes en nuestro medio. Por lo anterior serían de utilidad estudios enfocados en participantes occidentales.

Pese a no contarse con el grado de significancia estadísticas para establecer si las diferencias entre las tasas de EA serios reportadas para ambos grupos fueron significativas, impresionan ser relativamente frecuentes en ambos grupos y discretamente mayores en el grupo atezolizumab-bevacizumab. No obstante lo anterior, la calidad de vida reportada por los participantes y la SG fue superior en el grupo atezolizumab-bevacizumab. Asimismo, sería relevante identificar marcadores que permitan predecir y monitorizar la respuesta a atezolizumab-bevacizumab con el objetivo de aplicar esta terapia personalizadamente a cada paciente.

\section{Conclusión}

El estudio analizado permite concluir que en pacientes con $\mathrm{CHC}$ avanzado irresecable, la terapia con atezolizumab-bevacizumab es superior a sorafenib en términos de SG y SLP en una población seleccionada, caracterizada principalmente por función hepática preservada, sin complicaciones relevantes (Child-Pugh A) y buen estado funcional (ECOG 0-1). Además, esta terapia sería superior en términos de tasas de respuesta objetiva y de calidad de vida. No obstante, la certeza del beneficio categórico en comparación a la terapia estándar en algunos subgrupos, particularmente en aquellos con $\mathrm{CHC}$ de etiología no viral, es menor que en el resto de los participantes, dando cabida a nuevos estudios en poblaciones con estas características.

\section{Referencias}

1. Finn RS, Qin S, Ikeda M, Galle PR, Ducreux M, Kim TY, et al. Atezolizumab plus Bevacizumab in Unresectable Hepatocellular Carcinoma. N Engl J Med. 2020;382:1894-905.

2. Marrero JA, Kulik LM, Sirlin CB, Zhu AX, Finn RS, Abecassis MM, et al. Diagnosis, Staging, and Management of Hepatocellular Carcinoma: 2018 Practice Guidance by the American Association for the Study of Liver Diseases. Hepatology. 2018;68:723-50.

3. Ferlay J, Ervik M, Dikshit R, et al.
GLOBOCAN 2012 v1.0, Cancer Incidence and Mortality Worldwide: IARC CancerBase No. 11 [Internet]. International Agency for Research for Cancer; Lyon: France: 2013.

4. Kamińska M, Jankiewicz M, Kolak A, Budny B, et al. [Epidemiology and risk factors of hepatocellular carcinoma]. Pol Merkur Lekarski. 2017;43:133-9.

5. Dunbar JK, Dillon J, Garden OJ, Brewster DH. Increasing survival of hepatocellular carcinoma patients in Scotland: a review of national cancer registry data. HPB (Oxford). 2013;15:279-85.
6. Wiangnon S, Kamsa-ard S, Suwanrungruang K, Promthet S, Kamsaard S, Mahaweerawat S, et al. Trends in incidence of hepatocellular carcinoma, 1990-2009, Khon Kaen, Thailand. Asian Pac J Cancer Prev. 2012;13:1065-8.

7. Lau WY, Leung TW, Lai BS, Liew CT, Ho SK, Yu SC, et al. Preoperative systemic chemoimmunotherapy and sequential resection for unresectable hepatocellular carcinoma. Ann Surg. 2001;233:236-41.

8. Miller KD, Siegel RL, Lin CC, Mariotto AB, Kramer JL, Rowland JH, et al. Cancer treatment and survivorship 


\section{Medicina Basada en la evidencia en Gastroenterología}

statistics, 2016. CA Cancer J Clin. 2016;66:271-89.

9. Schacherer D, Schoelmerich J, ZuberJerger I. [The diagnostic approach to hepatocellular carcinoma]. Z Gastroenterol. 2007;45:1067-74.

10. Bruix J, Sherman M. Management of hepatocellular carcinoma: an update. Hepatology. 2011;53:1020-2.

11. Llovet JM, Ricci S, Mazzaferro V, Hilgard P, Gane E, Blanc JF, et al. Sorafenib in advanced hepatocellular carcinoma. N Engl J Med. 2008;359:37890.

12. Kudo M, Finn RS, Qin S, Han KH, Ikeda K, Piscaglia F, et al. Lenvatinib versus sorafenib in first-line treatment of patients with unresectable hepatocellular carcinoma: a randomised phase 3 noninferiority trial. Lancet. 2018;391:116373.

13. Cheng AL, Kang YK, Chen Z, Tsao CJ, Qin S, Kim JS, et al. Efficacy and safety of sorafenib in patients in the Asia-Pacific region with advanced hepatocellular carcinoma: a phase III randomised, double-blind, placebo-controlled trial. Lancet Oncol. 2009;10:25-34.

14. Lencioni R, Kudo M, Ye SL, Bronowicki JP, Chen XP, Dagher L, et al. GIDEON (Global Investigation of therapeutic DEcisions in hepatocellular carcinoma and $\mathrm{Of}$ its treatment with sorafeNib): second interim analysis. Int J Clin Pract. 2014;68:609-17.

15. Zhu AX, Finn RS, Edeline J, Cattan S, Ogasawara S, Palmer D, et al. Pembrolizumab in patients with advanced hepatocellular carcinoma previously treated with sorafenib (KEYNOTE-224): a non-randomised, open-label phase 2 trial. Lancet Oncol. 2018;19:940-52.

16. El-Khoueiry AB, Sangro B, Yau T, Crocenzi TS, Kudo M, Hsu C, et al. Nivolumab in patients with advanced hepatocellular carcinoma (CheckMate 040): an open-label, non-comparative, phase $1 / 2$ dose escalation and expansion trial. Lancet. 2017;389:2492-502.

17. Herbst RS, Soria JC, Kowanetz M, Fine GD, Hamid O, Gordon MS, et al.
Predictive correlates of response to the anti-PD-L1 antibody MPDL3280A in cancer patients. Nature. 2014;515:563-7.

18. Morse MA, Sun W, Kim R, He AR, Abada PB, Mynderse M, et al. The Role of Angiogenesis in Hepatocellular Carcinoma. Clin Cancer Res. 2019;25:912-20.

19. Finn RS, Bentley G, Britten CD, Amado R, Busuttil RW. Targeting vascular endothelial growth factor with the monoclonal antibody bevacizumab inhibits human hepatocellular carcinoma cells growing in an orthotopic mouse model. Liver Int 2009;29:284-90.

20. Motz GT, Santoro SP, Wang LP, Garrabrant T, Lastra RR, Hagemann IS, et al. Tumor endothelium FasL establishes a selective immune barrier promoting tolerance in tumors. Nat Med. 2014;20:607-15.

21. Voron T, Colussi O, Marcheteau E, Pernot S, Nizard M, Pointet AL, et al. VEGF-A modulates expression kof inhibitory checkpoints on CD8+ T cells in tumors. J Exp Med. 2015;212:139-48. 